\title{
Influence of Hip Fracture on Knee Pain During Postoperative Rehabilitation
}

\author{
Hee-Ju Kim, MD ${ }^{1}$, Seong Jae Lee, MD, PhD ${ }^{1}$, Jung Keun Hyun, MD, PhD ${ }^{1,2,3}$, \\ Seo-Young Kim, $\mathrm{MD}^{1}$, Tae Uk Kim, MD, $\mathrm{PhD}^{1}$ \\ ${ }^{1}$ Department of Rehabilitation Medicine, Dankook University College of Medicine, Cheonan; ${ }^{2}$ Department of Nanobiomedical \\ Science \& WCU Research Center, ${ }^{3}$ Institute of Tissue Regeneration Engineering, Dankook University, Cheonan, Korea
}

\begin{abstract}
Objective To investigate whether fracture type, surgical procedure, or fracture grade affect knee pain during postoperative rehabilitation after a hip fracture.

Methods We conducted a retrospective case-controlled study of 139 patients during postoperative rehabilitation after surgery for hip fractures. Patients were divided into two groups: patients experiencing knee pain during the first week of postoperative rehabilitation, and patients without knee pain. We compared the types of fracture, surgical procedure, and fracture grade between the two groups.

Results We enrolled 52 patients (37.4\%) with knee pain during the first weeks of postoperative rehabilitation. For type of fracture, knee pain was more common with intertrochanteric fracture than with femur neck fracture ( $48.8 \%$ vs. $21.1 \%$, respectively; $\mathrm{p}=0.001$ ). For the surgical procedure, there was no significant difference between the groups. For the fracture grade, the grades classified as unstable fractures were more common in the group of intertrochanteric fracture patients with knee pain than in those without knee pain $(74.1 \%$ vs. $36.4 \%$, respectively; $\mathrm{p}=0.002$ ).

Conclusion Intertrochanteric fracture affected knee pain after hip fracture surgery more than did femur neck fracture, particularly in unstable fractures. Furthermore, there was no difference in each fracture type according to the surgical procedure. Careful examination and management for knee pain is needed in patients with hip fracture surgery.
\end{abstract}

Keywords Hip fractures, Postoperative complications, Knee joint, Pain

\section{INTRODUCTION}

Hip fracture is a major health concern because of in- creased morbidity $[1,2]$, loss of independence [1], excessive deaths [2], and serious economic burden in the elderly population [3]. The incidence of hip fractures is

Received May 21, 2018; Accepted June 12, 2018

Corresponding author: Tae Uk Kim

Department of Rehabilitation Medicine, Dankook University College of Medicine, 201 Manghyang-ro, Dongnam-gu, Cheonan 31116, Korea. Tel: +8241-550-6641, Fax: +82-41-551-7062, E-mail: magnarbor@dankook.ac.kr

ORCID: Hee-Ju Kim (http://orcid.org/0000-0001-6877-5126); Seong Jae Lee (http://orcid.org/0000-0001-7867-4695); Jung Keun Hyun (http://orcid. org/0000-0001-9254-4424); Seo-Young Kim (http://orcid.org/0000-0002-5351-8044); Tae Uk Kim (http://orcid.org/0000-0001-9904-8781).

(c) This is an open-access article distributed under the terms of the Creative Commons Attribution Non-Commercial License (http://creativecommons.org/ licenses/by-nc/4.0) which permits unrestricted noncommercial use, distribution, and reproduction in any medium, provided the original work is properly cited. Copyright (c) 2018 by Korean Academy of Rehabilitation Medicine 
steadily rising. Although it is causing fewer deaths, there are still too many in Korea. Therefore, the socio-economic burden of hip fractures in Korea is expected to increase with increasing incidence [4].

Knee pain is an important factor in decreased walking ability and is a risk factor for falling $[5,6]$. Although many patients undergoing postoperative rehabilitation for a hip fracture have been treated for knee pain, few studies have investigated knee pain after hip fracture surgery. There are several studies on knee pain after intramedullary nailing surgery in a femur, but these studies included only those patients with intramedullary nailing after femur shaft fracture rather than hip fracture [7-13]. It is known that knee effusion occurs frequently after hip fracture [14-17], and previous reports suggest that knee joint stress during injury at the time of fracture or operation may be related to ipsilateral knee effusion $[16,17]$. Harato and Yoshida [15] suggest that knee effusion is associated with knee pain after hip fracture. Therefore, knee joint stress during injury at the time of fracture or operation may be associated with knee pain in patients undergoing postoperative rehabilitation.

It is expected that the type of fracture or surgical procedure resulting in excessive stress to the knee will more greatly affect knee pain. Harato and Yoshida [15] found that intertrochanteric fracture, which is categorized as an extracapsular fracture, is more associated with knee effusion than is intracapsular fracture. Based on this study, intertrochanteric fracture may be related to a higher incidence of knee pain after hip fracture than after intracapsular fracture. Pun et al. [16] suggested that there was no significant difference in the incidence of postoperative effusion according to the type of surgery. However, it is unclear whether this study completely excluded the effects of fracture types affecting knee effusion given the dearth of sufficient data. Moreover, Harato and Yoshida [15] found no significant difference in the incidence of postoperative effusion according to the severity of the fracture based on Evans or Garden classification. This contrasts to the hypothesis that knee pain increases with the severity of the fracture, which is believed to more greatly affect the knee. Evans classification of intertrochanteric fractures offers the best way to predict reliable anatomical reduction and to assess the risk of secondary fracture dislocation [18]; however, two studies have suggested that Evans classification has a poor reproduc- ibility $[19,20]$. The Arbeitsgemeinschaft für Osteosynthesefragen/Orthopaedic Trauma Association (AO/OTA) alphanumeric classification was developed because the previous classifications could not stratify valuable results regarding treatment options [21]. One study has suggested that its reliability and accuracy range from substantial to excellent [22]. Thus, in this study, we classified the intertrochanteric fractures according to AO/OTA alphanumeric classification and investigated its relationship with knee pain.

Considering the distance between the fracture site and the knee, as well as the protective effect of the capsule of the hip joint, knee pain may be more common in extracapsular fractures than in intracapsular fractures. Although there has been no study on the type of surgical procedure that produces more stress on the knee, cannulated screw fixation without intramedullary nailing process to the femur shaft, which would have affected the knee, is less likely to result in knee pain than are other surgical methods, such as proximal femoral nail antirotation (PFNA), hemiarthroplasty, and total hip replacement arthroplasty (THRA), for hip fracture. Moreover, it is assumed that the stress applied to the knee and the level of knee pain is related to the severity of the hip fracture.

To date, no research has addressed the effects of fracture types, surgical procedure, or fracture grade on knee pain itself, aside from knee effusion. Moreover, related studies have several limitations, as described above. We therefore aimed to investigate the effect of fracture type, surgical procedure, and fracture grade on knee pain in patients undergoing postoperative rehabilitation. We hypothesized that knee pain is more common in intertrochanteric fractures than in femur neck fractures, less common in cannulated screw fixation than in other surgical procedures for hip fracture, and more common in grades that more profoundly affect the knee.

\section{MATERIALS AND METHODS}

\section{Study subjects}

This retrospective case-controlled study was approved by the Dankook University Hospital Institutional Review Board (IRB No. 2017-07-011).

Patients who underwent hip fracture repair and who were referred to the rehabilitation department (RD) of Dankook University Hospital from January 1, 2011, to 
February 28, 2017, were screened. The inclusion criteria were (1) patients with intertrochanteric and femur neck fractures confirmed by a radiographic study and (2) patients who had surgery for the fracture.

The exclusion criteria were patients (1) with any knee pain during resting, or gait on the visual analog scale (VAS) of $10 \mathrm{~mm}$ or more in 3 months before hip fracture, (2) who had any operation history of the knee on the side of hip fracture, (3) who had severe postoperative complications, including pneumonia or delirium, that make evaluation of the knee impossible, (4) who had femoral and obturator neuropathy, and (5) who had another fracture of the leg on the side of the hip fracture.

A total of 139 patients (57 men and 82 women) with hip fracture were included. All patients were treated by the same orthopedic surgeon skilled in hip fracture surgery. The knee pain group included those patients with any subjective knee pain during resting, passive range of motion, weight bearing, or gait training on the VAS of $10 \mathrm{~mm}$ or more on the side of surgery during a week after the day of transfer to RD.

\section{Data collection}

Patient data were obtained from admission notes, progress notes, discharge summaries, operation records, doctor's order sheets, and radiologic records. Physical examination, including knee pain, is routinely performed on the day of transfer to the RD by physicians and recorded in the admission note. Thereafter, reevaluation was performed once a week and recorded in the progress note. Even if an evaluation was not conducted, patient complaints of symptoms were recorded in the progress notes.

\section{Data analysis}

Patients were divided into two groups, one of patients with knee pain, the other without knee pain. The baseline characteristics, including ambulatory status and pain medication at the time of transfer to $\mathrm{RD}$, were compared between the two groups. Ambulatory status was divided into three categories: bed ridden, wheel chair, and walker. Analgesics used included non-steroidal antiinflammatory drugs (NSAIDs), acetaminophen, tramadol, and muscle relaxant. Pain medication was divided into four categories: analgesics without muscle relaxant, muscle relaxant, muscle relaxant combined with other analgesics, and no medication. No opioids were used (except for tramadol) in this study. Muscle relaxants included orphenadrine and afloqualone. The types of fracture, surgical procedures, and grades of fracture were also compared between the groups. In an intertrochanteric fracture, the AO/OTA system was used to classify the grades of fractures. Types A1.1 to A2.1 were classified as a stable fracture, and types A2.2 to A3.3 were classified as unstable fractures [23]. In femur neck fractures, Garden classification was used to classify the grade of fractures. In this classification, stage 1 is an incomplete fracture (affected valgus fracture), stage 2 is a complete fracture without displacement, stage 3 is a complete fracture with partial displacement, and stage 4 is a complete fracture with full displacement [24].

\section{Statistical analysis}

Baseline characteristics of the two groups (with or without knee pain) were compared by the independent ttest for continuous variables, and by the chi-square test or Fisher exact test for categorical variables. Outcome measures were compared using the chi-square test or Fisher exact test. Further, using the type of fracture as an independent variable and the knee pain as a dependent variable, a multivariate logistic regression analysis was performed to calculate odds ratio. Adjustments were made for age, sex, operation time, anesthesia time, and body mass index. The values are presented as the mean \pm standard deviation or number of patients (\%). Statistical significance was set at $\mathrm{p}<0.05$. All statistical analyses were performed using SPSS Statistics version 20.0 for Windows (IBM, New York, NY, USA).

\section{RESULTS}

\section{Patients}

We assessed 209 patients for eligibility. There were 39 who did not meet the inclusion criteria. We excluded those who had any knee pain on the VAS of $10 \mathrm{~mm}$ or more in the 3 months before hip fracture surgery (21 patients), a history of knee operation on the side of the hip fracture ( 2 patients); severe postoperative complications including pneumonia or delirium (5 patients); femoral and obturator neuropathy (1 patient); and combined fractures of the leg on the side of hip ( 2 patients). Thus a total of 139 patients were included in the final analysis. 
The incidence of knee pain was 37.4\% (52 of 139 patients). Of all 139 patients, 49 had PFNA, 33 had hemiarthroplasty for intertrochanteric fracture, 39 had hemiarthroplasty, 11 had THRA, and 7 had cannulated screw fixation for femur neck fracture.

\section{Baseline characteristics}

The demographics of the patients in both groups are shown in Table 1. Age was similar between the groups with and without knee pain $(72.1 \pm 10.2$ vs. $73.0 \pm 13.0$ years, respectively; $\mathrm{p}=0.121$ independent t-test). Sex, body mass index (BMI), laterality, operation time, anesthesia time, postoperative days (POD), ambulatory status, and pain medication at the time of transfer to the $\mathrm{RD}$ were also not statistically different between the groups. But hospital stay in RD was significantly longer for the group with knee pain than for the other group $(21.2 \pm 9.7$ vs. $16.1 \pm 9.7$ days, respectively; $\mathrm{p}=0.003$ independent $\mathrm{t}$ - test).

\section{Types of fracture}

Incidence of knee pain was higher in the group with intertrochanteric fractures than in the group with femur neck fractures ( $48.8 \%$ vs. $21.1 \%$, respectively; $\mathrm{p}=0.001$ ) (Table 2). Multivariate logistic regression analysis was conducted with age, sex, operation time, anesthesia time, and BMI. Knee pain after hip fracture surgery was 1.342 times more likely to occur with intertrochanteric fractures than with femur neck fractures (Table 3).

\section{Surgical procedures}

For the surgical procedures, there was no significant difference in the incidence of knee pain between the group of patients with PFNA and hemiarthroplasty for intertrochanteric fractures $(57.1 \%$ vs. $36.4 \%$, respectively; $\mathrm{p}=0.065$ ), and was similar for the groups of patients with

Table 1. Baseline characteristics of study subjects

\begin{tabular}{|c|c|c|c|}
\hline & $\begin{array}{c}\text { Knee pain }(-) \\
(\mathbf{n}=87)\end{array}$ & $\begin{array}{c}\text { Knee pain }(+) \\
\quad(n=52)\end{array}$ & p-value \\
\hline Age (yr) & $76.1 \pm 10.2$ & $73.0 \pm 13.0$ & $0.121^{\mathrm{a})}$ \\
\hline Sex & & & $0.236^{\mathrm{b})}$ \\
\hline Male & 39 & 18 & \\
\hline Female & 48 & 34 & \\
\hline BMI, $>25 \mathrm{~kg} / \mathrm{m}^{2}$ & $17(19.8)$ & $10(19.2)$ & $0.911^{\mathrm{b})}$ \\
\hline Laterality & & & $0.939^{\mathrm{b})}$ \\
\hline Left & 46 & 28 & \\
\hline Right & 41 & 24 & \\
\hline POD at transfer to $\mathrm{RD}$ & $10.7 \pm 4.9$ & $10.5 \pm 6.3$ & $0.866^{a)}$ \\
\hline Operation time (min) & $85.2 \pm 31.7$ & $89.2 \pm 27.0$ & $0.453^{\mathrm{a})}$ \\
\hline Anesthesia time (min) & $152.1 \pm 35.6$ & $159.2 \pm 29.9$ & $0.227^{\mathrm{a})}$ \\
\hline Ambulatory status at transfer & & & $0.286^{\mathrm{c})}$ \\
\hline Bed ridden & $71(81.6)$ & $47(90.4)$ & \\
\hline Wheelchair & $13(14.9)$ & $5(9.6)$ & \\
\hline Walker & $3(3.4)$ & $0(0.0)$ & \\
\hline Pain medication & & & $0.271^{c)}$ \\
\hline Analgesics without muscle relaxant & $16(18.4)$ & $14(26.9)$ & \\
\hline Muscle relaxant & $4(4.6)$ & $0(0.0)$ & \\
\hline Analgesics with muscle relaxant & $44(50.6)$ & $28(53.8)$ & \\
\hline No medication & $23(26.4)$ & $10(19.2)$ & \\
\hline Total length of stay in RD (day) & $16.1 \pm 9.7$ & $21.2 \pm 9.7$ & $0.003^{\mathrm{a})}$ \\
\hline
\end{tabular}

Values are presented as the mean \pm standard deviation or number of patients (\%). $\mathrm{BMI}$, body mass index; POD, postoperative days; RD, rehabilitation department.

${ }^{\text {a) }}$ Independent t-test, ${ }^{\mathrm{b})}$ chi-square test, ${ }^{\mathrm{c}}$ Fisher exact test. 
hemiarthroplasty, THRA, and cannulated screw for femur neck fracture $(20.5 \%$ vs. $27.3 \%$ vs. $14.3 \%$, respectively; $\mathrm{p}=0.885$ ) (Table 4).

\section{Fracture grade}

In the group of patients with intertrochanteric fractures, knee pain was more common in grades classified as unstable fractures than in stable fractures $(74.1 \%$ vs. $36.4 \%$, respectively; $\mathrm{p}=0.001$ ) (Table 5). The AO/OTA classification was used to classify the grade. Types A1.1 to A2.1 were classified as stable fractures, and Types A2.2 to A3.3 were classified as unstable fractures [23]. Fig. 1 shows that, as the grade increases, the proportion of patients with knee pain increases. However, in the group of patients with femur neck fractures, there was no significant difference in the incidence of knee pain according to the fracture grades classified by Garden classification.

\section{DISCUSSION}

This study investigated whether fracture type, surgical procedure, or fracture grade affected knee pain during postoperative rehabilitation after hip fracture. Our results demonstrated that the incidence of knee pain in patients undergoing rehabilitation after hip fracture surgery is as high as $37.4 \%$ (52 of 139 patients). Since every patient had pain medication to control postoperative pain before being transferred to the $\mathrm{RD}$, the incidence of knee pain may

Table 2. Comparison of type of fracture in patients with and without knee pain

\begin{tabular}{lccc}
\hline & $\begin{array}{c}\text { Knee pain } \\
(-)\end{array}$ & $\begin{array}{c}\text { Knee pain } \\
(+)\end{array}$ & $\begin{array}{c}\text { p- } \\
\text { value }^{\text {a) }}\end{array}$ \\
\hline Intertrochanteric fracture & $42(51.2)$ & $40(48.8)$ & 0.001 \\
Femur neck fracture & $45(78.9)$ & $12(21.1)$ & \\
\hline
\end{tabular}

Values are presented as number of patients (\%).

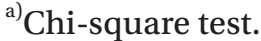

be higher than this result. Knee pain was more common in the group with intertrochanteric fractures than for those with femur neck fractures. However, for the surgical procedures, there was no significant difference between the groups. In the group with intertrochanteric fractures, knee pain was more common for unstable fracture than for stable fracture.

This study suggests that knee pain was more common in the group with intertrochanteric fractures than for those with femur neck fractures. Hip fractures are classified as either intracapsular or extracapsular. Intertrochanteric fractures are categorized as extracapsular, whereas femur neck fractures are categorized as intracapsular fractures [25]. These results are consistent with our hypothesis and suggest that, when hip fracture occurs, the joint capsule and synovial fluid appear to have a shock-absorbing effect in reducing abnormal stress transmitted to the knee.

Pun et al. [16] hypothesized that, during surgery, the twist and traction force applied to the limb and the impact and vibration transmitted to the knee during the reaming procedure would be an important factor in knee

Table 4. Comparison of type of surgery in patients with and without knee pain

\begin{tabular}{|c|c|c|c|}
\hline & $\begin{array}{c}\text { Knee pain } \\
(-)\end{array}$ & $\begin{array}{c}\text { Knee pain } \\
(+)\end{array}$ & $\begin{array}{c}\text { p- } \\
\text { value }^{\text {a) }}\end{array}$ \\
\hline Intertrochanteric fracture & & & $0.065^{\mathrm{a})}$ \\
\hline PFNA & $21(42.9)$ & $28(57.1)$ & \\
\hline Hemiarthroplasty & $21(63.6)$ & $12(36.4)$ & \\
\hline Femur neck fracture & & & $0.885^{\mathrm{b})}$ \\
\hline Hemiarthroplasty & $31(79.5)$ & $8(20.5)$ & \\
\hline THRA & $8(72.7)$ & $3(27.3)$ & \\
\hline Cannulated screw & $6(85.7)$ & $1(14.3)$ & \\
\hline
\end{tabular}

Values are presented as number of patients (\%).

PFNA, proximal femoral nail antirotation; THRA, total hip replacement arthroplasty.

${ }^{a)}$ Chi-square test, ${ }^{\text {b) }}$ Fishers exact test.

Table 3. OR and CI of knee pain according to types of fracture

\begin{tabular}{lccccc}
\hline & \multicolumn{2}{c}{ Crude } & \multicolumn{2}{c}{ Adjusted $^{\text {a) }}$} \\
\cline { 2 - 3 } \cline { 5 - 6 } & OR (95\% CI) & p-value & & OR (95\% CI) & p-value \\
\hline Femur neck fracture & 1.00 & - & & 1.00 & - \\
Intertrochanteric fracture & $1.27(1.65-7.71)$ & 0.001 & & $1.34(1.75-8.37)$ & 0.001 \\
\hline
\end{tabular}

OR, odd ratio; $\mathrm{CI}$, confidence interval.

${ }^{\text {a) }} \mathrm{OR}$ and $95 \% \mathrm{CI}$ were estimated by logistic regression analysis adjusted for age, sex, operation time, anesthesia time, and body mass index. 
Table 5. Comparison of stability of intertrochanteric fracture in patients with and without knee pain

\begin{tabular}{lccc}
\hline & $\begin{array}{c}\text { Knee pain } \\
(-)\end{array}$ & $\begin{array}{c}\text { Knee pain } \\
(+)\end{array}$ & p-value $^{\text {a) }}$ \\
\hline Stable fracture & $35(63.6)$ & $20(36.4)$ & 0.001 \\
Unstable fracture & $7(25.9)$ & $20(74.1)$ & \\
\hline
\end{tabular}

Values are presented as number of patients (\%).

${ }^{\text {a) }}$ Chi-square test.

effusion, but there was no statistically significant difference in the incidence of postoperative effusion in the knee in terms of the surgical procedure. These results are consistent with our study, which showed no difference in the incidence of knee pain correlated with the surgical procedures. Cannulated screw fixation without intramedullary nailing process to the femur shaft is less likely to result in knee pain than are other types of surgery. However, based on the studies of Pun et al. [16] and ours, the incidence of knee pain is not meaningfully related to the surgical procedure.

In the group of patients with intertrochanteric fractures, a type of extracapsular fracture, unstable fractures were more common in the group with knee pain than in the group without knee pain. Furthermore, as the grade increased, the proportion of patients with knee pain increased (Fig. 1). This differs from the results of Harato and Toshida [15], who did not find any difference in the incidence of postoperative effusion related to grade of fracture [6]. We used the AO/OTA system to classify the intertrochanteric fractures, whereas Harato and Toshida [15] used Evan classification of intertrochanteric fractures, which offers the best way to predict the possibility of obtaining reliable anatomical reduction and to assess the risk of secondary fracture dislocation [18]. However, the two studies suggest that Evan classification has poor reproducibility $[19,20]$. One study found that the AO/OTA system is reliable and has an accuracy that ranges from substantial to excellent [22]. In the AO/OTA system, the number of fracture fragments and incidence of more distal fractures increase in proportion with the grade; hence a higher the grade indicates a more profound impact on the knee, or a fracture that is closer to the knee. Our results are acceptable, because the AO/OTA classification is more reliable than the taxonomy used in previous studies, and it matches our reasonable hypothesis that more shock will be transmitted to the knee by a more severe

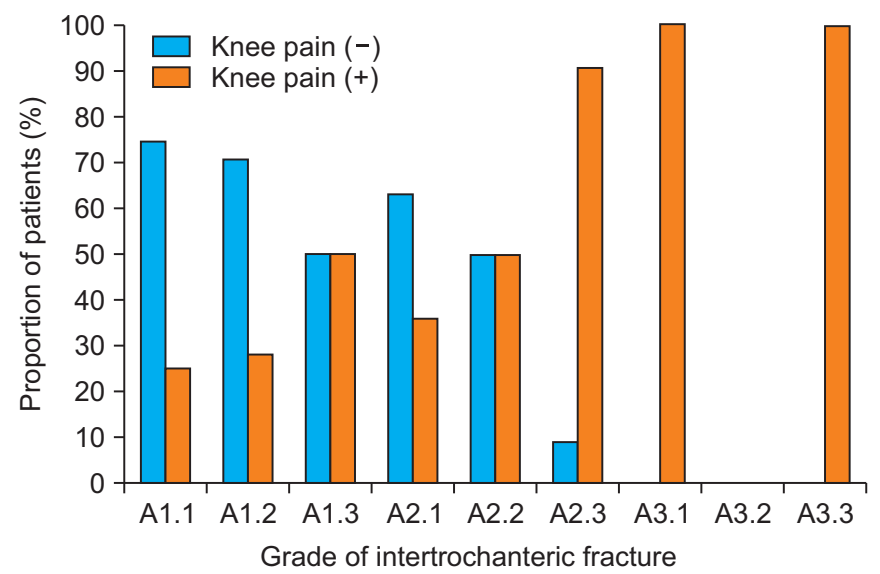

Fig. 1. Proportion of patients with and without knee pain according to grade of intertrochanteric fracture. AO/OTA alphanumeric classification was used to classify intertrochanteric fractures. The proportion of patients with knee pain increases in proportion to the grade. AO/OTA, Arbeitsgemeinschaft für Osteosynthesefragen/Orthopaedic Trauma Association.

fracture, which ultimately results in greater knee pain. In the group of patients with femur neck fractures, a type of intracapsular fracture, there was no significant difference in the incidence of knee pain associated with the grade of fracture. Unlike intertrochanteric fractures, which are extracapsular fracture, there was no correlation of grade with knee pain in the femur neck fracture, which is an intracapsular fracture. These results also suggest that the joint capsule and synovial fluid in the hip joint have a buffering effect that mitigates the amount of shock delivered to the knee in the intracapsular fracture.

Four studies have provided information about the localization and etiology of the knee pain in antegrade femoral nailing for femur shaft fracture. These studies suggest that the presence of a locking screw and implant in the medullary canal is one cause of knee pain after surgery $[8-10,12]$. However, this does not explain the cause of knee pain in this study. Unlike cases of femurshaft fracture, patients in this study had a locking screw and/or implant at a considerable distance from the knee. There is one case report that assessed other possibilities for the cause of knee pain after hip fractures. One such case presenting as isolated knee pain has been reported. Guss speculated that the anatomy of hip joint innervation accounts for this clinical presentation. The hip joint is innervated by nerves derived from branches of the obturator and femoral nerves. Branches of these same 
nerves also innervate structures around the distal thigh and knee. Innervation of the hip joint may be another etiology of knee pain after hip fractures [26]. However, if this innervation is a main cause, more knee pain may have been observed in intracapsular fractures, which are thought to more greatly affect the hip joint considering the anatomy of the hip.

Some limitations of this study need to be discussed. First, it did not include patients with subtrochanteric and femoral head fractures, because there were few such patients. During the study period, there were only six patients with subtrochanteric fractures, and none with femoral head fractures. In patients with subtrochanteric fracture, 5 out of 6 knee pain. Such a high incidence of knee pain is also consistent with our finding that knee pain is more common in extracapsular fractures than in intracapsular fractures. Second, adequate evaluation of knee pain was not performed before surgery. When hip fracture occurs, it is difficult for the patient to accurately recognize knee pain because it is masked by the severe pain at the fracture site and by medication for the pain. Third, all patients were not evaluated by knee plain radiographs. Previous studies have suggested that radiographic knee osteoarthritis (Kellgren-Lawrence grade of $\geq 2$ ) is associated with knee effusion [15]. This may be another risk factor for knee pain after hip fracture, but it is costly to perform routine knee plain radiography in all patients with hip fracture in order to evaluate a risk factor for knee pain. Last, there was no long-term follow-up for knee pain, and the effect of knee pain on the patient's functional outcomes was not studied. In the study of knee effusion after hip fracture surgery, knee effusion improved after 6 weeks [17] and there was no significant difference in the level of walking ability between patients with and without knee effusion at 6 weeks [16]. However, our result suggests that patients with knee pain have a longer hospital stay in RD than do patients without knee pain. Moreover, secondary hip fractures are a serious problem in patients with hip fracture surgery [27-29], and knee pain has been reported to be an important risk factor for multiple falls by women [30]. Therefore, knee pain after hip fracture surgery may be an important problem. Further research is needed to discover the etiology and effective management of knee pain after hip fracture surgery along with its effect on the patient's functional outcomes.
In conclusion, intertrochanteric fracture had a greater effect on knee pain after hip fracture surgery than did femur neck fracture, particularly in unstable fractures. Moreover, there was no difference in each type of fracture according to the surgical procedures. The incidence of knee pain in patients undergoing rehabilitation after hip fracture surgery is not so low that it can be overlooked. Careful examination and management for knee pain are needed for patients who have undergone hip fracture surgery.

\section{CONFLICT OF INTEREST}

No potential conflict of interest relevant to this article was reported.

\section{REFERENCES}

1. Lee SR, Ha YC, Kang H, Park YG, Nam KW, Kim SR. Morbidity and mortality in Jeju residents over 50 -years of age with hip fracture with mean 6 -year follow-up: a prospective cohort study. J Korean Med Sci 2013;28:1089-94.

2. Yoon HK, Park C, Jang S, Jang S, Lee YK, Ha YC. Incidence and mortality following hip fracture in Korea. J Korean Med Sci 2011;26:1087-92.

3. Choi HJ, Shin CS, Ha YC, Jang S, Jang S, Park C, et al. Burden of osteoporosis in adults in Korea: a national health insurance database study. J Bone Miner Metab 2012;30:54-8.

4. Ha YC, Kim TY, Lee A, Lee YK, Kim HY, Kim JH, et al. Current trends and future projections of hip fracture in South Korea using nationwide claims data. Osteoporos Int 2016;27:2603-9.

5. Muraki S, Akune T, Oka H, En-Yo Y, Yoshida M, Nakamura $\mathrm{K}$, et al. Prevalence of falls and the association with knee osteoarthritis and lumbar spondylosis as well as knee and lower back pain in Japanese men and women. Arthritis Care Res (Hoboken) 2011;63:142531.

6. Muraki S, Akune T, Oka H, Ishimoto Y, Nagata K, Yoshida $\mathrm{M}$, et al. Physical performance, bone and joint diseases, and incidence of falls in Japanese men and women: a longitudinal cohort study. Osteoporos Int 2013;24:459-66.

7. Harris I, Hatfield A, Donald G, Walton J. Outcome af- 
ter intramedullary nailing of femoral shaft fractures. ANZ J Surg 2003;73:387-9.

8. Wolinsky PR, McCarty E, Shyr Y, Johnson K. Reamed intramedullary nailing of the femur: 551 cases. J Trauma 1999;46:392-9.

9. Braten M, Terjesen T, Rossvoll I. Femoral shaft fractures treated by intramedullary nailing: a follow-up study focusing on problems related to the method. Injury 1995;26:379-83.

10. Ostrum RF, Agarwal A, Lakatos R, Poka A. Prospective comparison of retrograde and antegrade femoral intramedullary nailing. J Orthop Trauma 2000;14:496501.

11. Ricci WM, Bellabarba C, Evanoff B, Herscovici D, DiPasquale T, Sanders R. Retrograde versus antegrade nailing of femoral shaft fractures. J Orthop Trauma 2001;15:161-9.

12. Toms AD, Morgan-Jones RL, Spencer-Jones R. Intramedullary femoral nailing: removing the nail improves subjective outcome. Injury 2002;33:247-9.

13. Tornetta P 3rd, Tiburzi D. Antegrade or retrograde reamed femoral nailing: a prospective, randomised trial. J Bone Joint Surg Br 2000;82:652-4.

14. Christodoulou AG, Givissis P, Antonarakos PD, Petsatodis GE, Hatzokos I, Pournaras JD. Knee joint effusion following ipsilateral hip surgery. J Orthop Surg (Hong Kong) 2010;18:309-11.

15. Harato K, Yoshida H. Pseudogout at the knee joint will frequently occur after hip fracture and lead to the knee pain in the early postoperative period. J Orthop Surg Res 2015;10:4.

16. Pun WK, Chow SP, Chan KC, Ip FK, Leong JC. Effusions in the knee in elderly patients who were operated on for fracture of the hip. J Bone Joint Surg Am 1988;70:117-8.

17. Shahid MS, Murphy D, O’Donnell T, Ryan E, Kaliszer $\mathrm{MM}$, Masterson E. A prospective study for evaluation of knee effusion after hip surgery. Ir Med J 2002;95:140-1.

18. Jensen JS. Classification of trochanteric fractures. Acta Orthop Scand 1980;51:803-10.

19. Andersen E, Jorgensen LG, Hededam LT. Evans' clas- sification of trochanteric fractures: an assessment of the interobserver and intraobserver reliability. Injury 1990;21:377-8.

20. Gehrchen PM, Nielsen JO, Olesen B. Poor reproducibility of Evans' classification of the trochanteric fracture: assessment of 4 observers in 52 cases. Acta Orthop Scand 1993;64:71-2.

21. Ahn H, Court-Brown CM, McQueen MM, Schemitsch $\mathrm{EH}$. The use of hospital registries in orthopaedic surgery. J Bone Joint Surg Am 2009;91 Suppl 3:68-72.

22. Meling T, Harboe K, Enoksen $\mathrm{CH}$, Aarflot M, Arthursson AJ, Soreide K. How reliable and accurate is the AO/OTA comprehensive classification for adult long-bone fractures? J Trauma Acute Care Surg 2012;73:224-31.

23. Sonawane DV. Classifications of intertrochanteric fractures and their clinical importance. Trauma Int 2015;1:7-11.

24. Frandsen PA, Andersen E, Madsen F, Skjodt T. Garden's classification of femoral neck fractures: an assessment of inter-observer variation. J Bone Joint Surg Br 1988;70:588-90.

25. Mears SC. Classification and surgical approaches to hip fractures for nonsurgeons. Clin Geriatr Med 2014;30:229-41.

26. Guss DA. Hip fracture presenting as isolated knee pain. Ann Emerg Med 1997;29:418-20.

27. Mitani S, Shimizu M, Abo M, Hagino H, Kurozawa Y. Risk factors for second hip fractures among elderly patients. J Orthop Sci 2010;15:192-7.

28. Omsland TK, Emaus N, Tell GS, Ahmed LA, Center JR, Nguyen ND, et al. Ten-year risk of second hip fracture: a NOREPOS study. Bone 2013;52:493-7.

29. Yamanashi A, Yamazaki K, Kanamori M, Mochizuki K, Okamoto S, Koide Y, et al. Assessment of risk factors for second hip fractures in Japanese elderly. Osteoporos Int 2005;16:1239-46.

30. Muraki S, Akune T, Ishimoto Y, Nagata K, Yoshida M, Tanaka S, et al. Risk factors for falls in a longitudinal population-based cohort study of Japanese men and women: the ROAD Study. Bone 2013;52:516-23. 